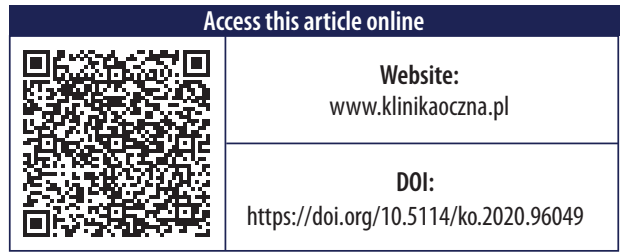

\title{
Can optic nerve sheath meningioma affect the post-operative course after trabeculectomy? - case report
}

\author{
Michał Post, Magdalena Durajczyk, Wojciech Lubiński \\ $2^{\text {nd }}$ Chair and Department of Ophthalmology, Pomeranian Medical University in Szczecin, Poland
}

\begin{abstract}
The authors report a case of a 37-year-old man treated due to concomitant open-angle glaucoma and chronic optic disc oedema in his left eye. During 4-year observation the patient underwent routine glaucoma treatment and periocular triamcinolone injections, with periodical resolution of symptoms (visual snow) and improvement in the eye electrophysiological performance. During diagnostic proceeding, including routine ophthalmological examinations and extensive imaging and electrophysiological examinations (CT, MRI, angioMRI), left-sided optic nerve sheath meningioma was diagnosed. Due to intolerance of topical medicines and fluctuation of intraocular pressure, trabeculectomy with mitomycin was performed
\end{abstract}

in the patient, complicated with chronic choroidal effusion and hypotony maculopathy. In the discussion, the authors analyse the potentially adverse effect of optic nerve sheath meningioma on the persistent nature of post-operative complications. The trabeculectomy procedure in this group of patients seems to be increasedrisk surgery and is not recommended. Therefore in patients with optic nerve sheath meningioma procedures associated with lower risk of post-operative hypotony (minimally invasive glaucoma surgery (MIGS), valves) should be considered.

KEY WORDS: optic nerve sheath meningioma, glaucoma, hypotony, trabeculectomy, hypotony maculopathy.

\section{INTRODUCTION}

Optic nerve sheath meningioma (ONSM) is a rare, benign tumour originating from meningothelial cells of the meninges surrounding the optic nerve. It represents $1 / 3$ of primary optic nerve tumours [1]. Over $90 \%$ of ONSM cases are secondary to central nervous system (CNS) tumours $[2,3]$. Optic nerve sheath meningioma, despite being considered a benign tumour, results in gradual deterioration of vision, due to compression of the optic nerve and vessels supplying it. The tumour is usually unilateral (95\%) and is three times more common in women than in men [2]. Mean age of onset of the disease is 41 years. Only $4 \%$ of optic nerve sheath meningiomas occur at the age of $<20$ years, according to Dutton. A set of symptoms, known as the Hoyt-Spencer triad, is typical for ONSM: 1) painless, gradual deterioration of vision, 2) atrophy/oedema of optic disc, 3) retino-ciliary leakage.

Optic nerve sheath meningioma may cause visual field defects of any type and affect the appearance of the optic disc. This is particularly important in patients with chronic optic nerve diseases, e.g. glaucoma. To date, there is only one case of concomitant optic nerve sheath meningioma resulting in progression of visual field defects in a glaucoma pa- tient described in the literature [4]. In this paper the authors present, for the first time, the case of an ONSM patient who underwent the trabeculectomy procedure complicated with chronic hypotony. The authors analyse the potential effect of ONSM on the course of glaucoma and post-trabeculectomy complications.

\section{CASE PRESENTATION}

The 33-year-old man presented to an ophthalmologist with symptoms of visual snow, occurring as a dozen or so episodes daily. To date, the patient had not suffered from any general or ophthalmological diseases, and had not used any medicines or drugs. Initial examination revealed regular visual acuity (V OD/OS 1.0) and increased intraocular pressure (IOP) OD $34.7 \mathrm{mmHg}$, OS $29.2 \mathrm{mmHg}$. Appearance of the anterior segment was normal in both eyes. Right eye fundus was unremarkable, while in the left eye features of chronic optic disc oedema, folds of internal retinal layers in the posterior pole and venous dilatation were noted (Figure 1). Optical coherence tomography (OCT) of the left eye revealed oedema of the optic nerve head with marked thickening of the retinal nerve fibre layer (RNFL (Figure 2A) and retinal folds in the nasal 
part of the macula (Figure 2B). Laboratory tests and imaging examinations of the chest and abdominal cavity ruled out inflammatory and ischaemic aetiology of the optic disc oedema. Fluorescein angiography revealed papilloedema, flame-shaped haemorrhages around the disc, dilated optic nerve capillaries and increasing hyperfluorescence. Pattern visual evoked potentials (PVEP) examination performed according to International Society for Clinical Electrophysiology of Vision (ISCEV) standards revealed such abnormalities as reduction of amplitude and prolonged latency of P100 wave in the left eye. In the statistical visual field (PS24/2, Humphrey), decreased retinal sensitivity (mean deviation $[\mathrm{MD}]-2.94 \mathrm{~dB}$, pattern standard deviation [PSD] $3.74 \mathrm{~dB}$ ) and paracentral arcuate defect in the upper hemisphere in the left eye were found (Figure 3). Diagnostic procedures performed in the patient during the first year included computed tomography (CT) examination, magnetic resonance imaging (MRI) of head and orbits performed twice and angioMRI of orbits. Interestingly, none of these examination revealed features of optic nerve sheath meningioma. The patient was monitored by a specialist in optic nerve disease, who excluded optic nerve drusen and infectious and non-infectious causes of optic disc oedema. Therefore, the patient was diagnosed with secondary open-angle glaucoma with idiopathic optic disc oedema in the left eye and ocular hypertension in the right eye.

Prostaglandin monotherapy was applied in the patient, and after several months was replaced with doublet pharmacotherapy in both eyes. In the third year of observation laser trabeculoplasty was performed. Due to significantly increased visual snow symptoms in the left eye, the decision was made to administer $40 \mathrm{mg}$ of triamcinolone acetate (Kenalog) periocularly in the left eye. Eventually, the patient received five injections during three years. After each injection the patient reported subjective improvement in visual acuity and partial resolution of visual snow symptoms. The Kenalog injections had no significant effect on the patient's visual field, but in PVEP examination some improvement in left optic nerve function was noted in the form of increase in amplitude of P100 wave. In the third year of observation third MRI of orbits was performed and revealed contrast enhancement of the left optic nerve sheath with irregular thickening up to $2 \mathrm{~mm}$ (Figure 4A). On the basis of the MRI picture, left-sided optic nerve sheath meningioma was diagnosed (Figure 4B). Simultaneously, in the third year of observation intolerance of topical medicines (contact conjunctivitis) and increases of intraocular pressure in the left eye up to $36 \mathrm{mmHg}$ were noted. Most likely they were secondary to the steroid injections. The patient still had complete visual acuity (V OS 1.0) and no progression in visual field defect.

Due to uncontrolled IOP increases and intolerance of topical medicines, trabeculectomy with mitomycin $(0.05 \%$ for 3 minutes) was performed in the patient. On the $1^{\text {st }}$ day after the procedure V OS 1/50 and hypotony were noted. Due to leakage from the surgical conjunctival wound (Seidel test positive) additional conjunctival sutures and a contact lens were applied. In subsequent weeks of observation, ante-

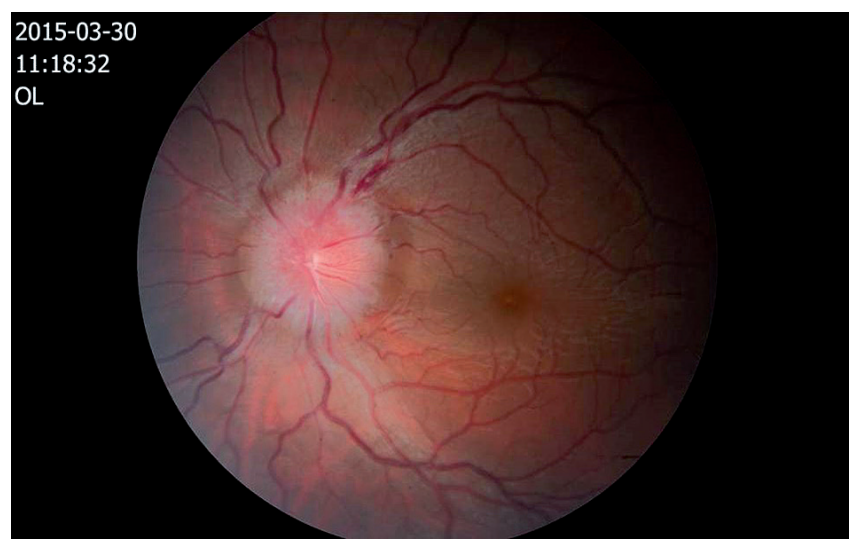

Figure 1. Image of the fundus at the time of diagnosis - swelling of the optic disc, venous stasis, tortuous veins, retinal folds

rior chamber shallowing and excessive filtration of the fistula, without leakage, were noted. On the eye fundus, retinal and choroidal folds (OCT scan - Figure 2D), hypotony maculopathy, increased optic disc oedema (OCT scan - Figure 2C) and choroidal effusion were found (Figure 5). In subsequent weeks intraocular pressure was normalised; however, the appearance of left eye fundus did not change significantly during further observation. Eventually, the patient's visual acuity decreased to the V OS hand motion (HM) level, with normal IOP.

The patient is under constant ophthalmological and neurological care. Currently he does not require surgery or radiation therapy for ONSM in the left eye. The right eye is currently in a stable condition with IOP $<18 \mathrm{mmHg}$. After switching drops to preservative-free ones (dorzolamide + timolol) irritation symptoms decreased. No further surgical intervention is planned in the right eye.

\section{DISCUSSION}

Optic nerve sheath meningioma constitutes 1-2\% of all meningioma cases. The primary symptom of ONSM is painless deterioration of vision, which may be accompanied with relative afferent pupillary defect (RAPD) [5]. Mean time from the onset of symptoms to diagnosis is 12 months (0-216 months) for primary ONSM, and 6 months in the case of tumours secondary to CNS meningioma (90\%) [5]. The diagnosis is based on physical (ophthalmologic) examination and imaging examinations (histopathology examination is not required). Typical appearance of ONSM includes tubular calcifications visible in orbital MRI - "tram-track" sign present in $20-50 \%$ of cases. In the case in question, optic nerve lesions were not detected until the third MRI, which delayed the correct diagnosis by 3 years. In the case of primary tumours, optic disc oedema is present in almost half of them, and optic disc atrophy in another half, while normal appearance of the disc is rare [5]. Visual acuity in ONSM is variable. According to Dutton, only $24 \%$ of patients had visual acuity at the counting fingers (CF) level or lower. Almost half of patients have visual acuity $>0.5[5,6]$. In the case in question, visual acuity was stable in 3-year observation (V OS 1.0) and deteriorated only due to post-operative complications. ONSM may cause visual field defects of various types. According to 

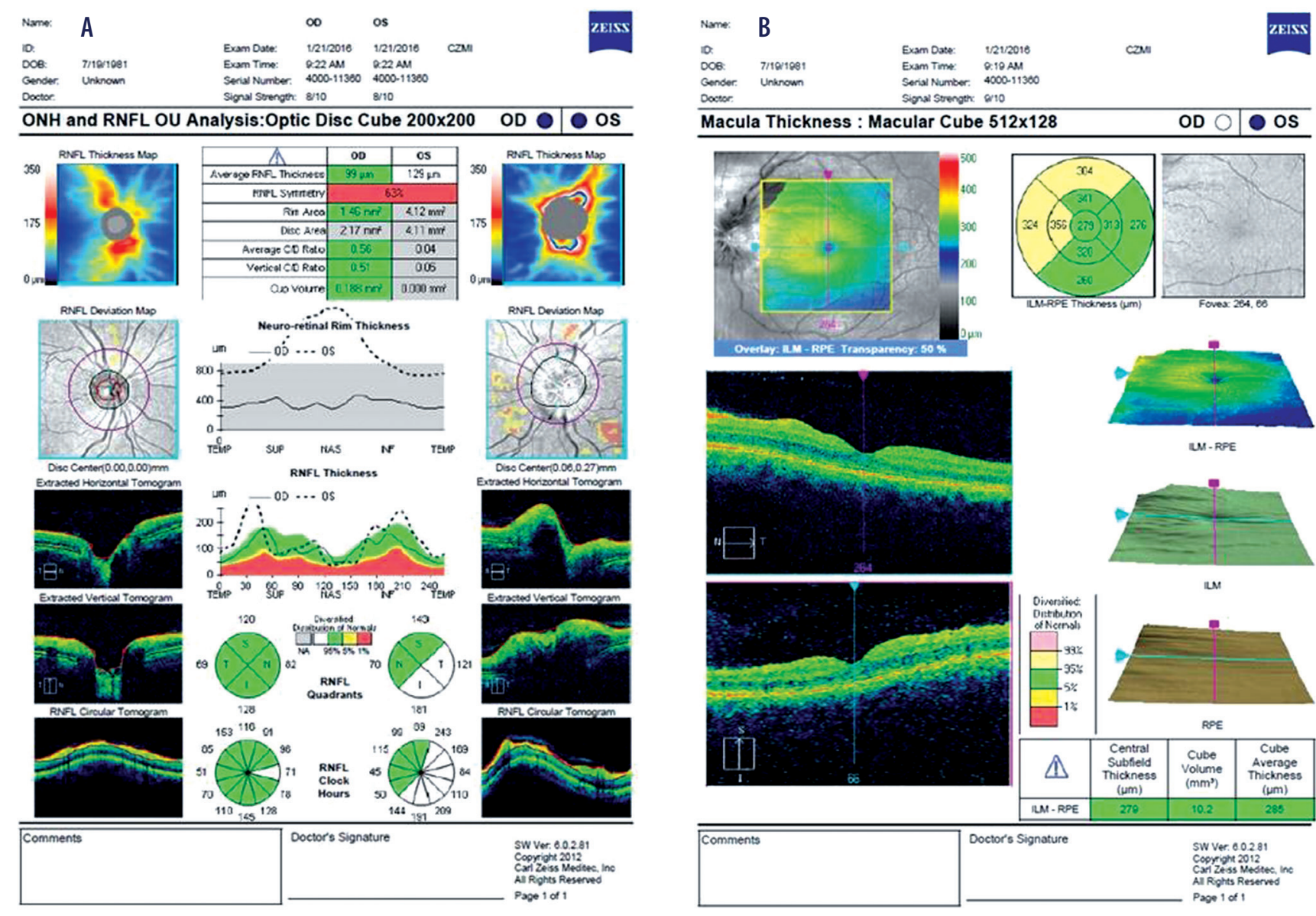

\begin{tabular}{ll|l}
\hline Macula Thickness : Macular Cube 512x128 & OD O & $\bigcirc$ OS \\
\hline
\end{tabular}
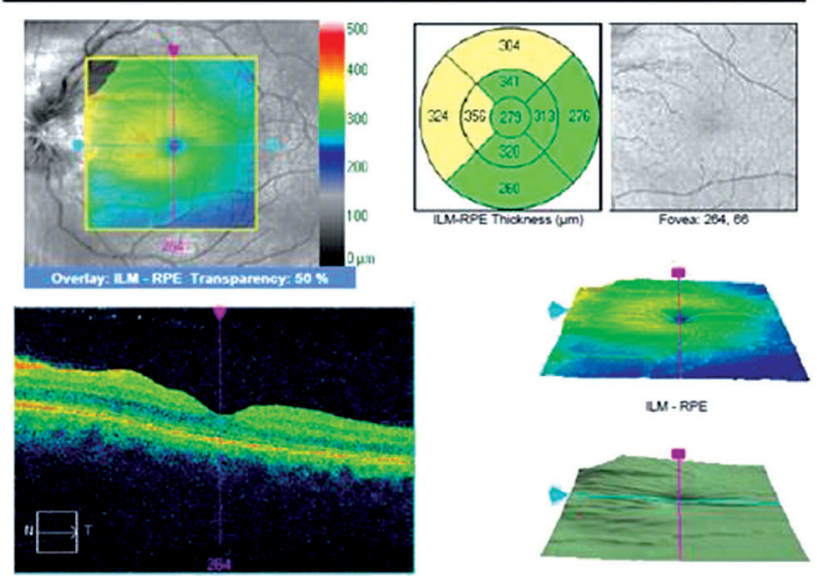

UM-RPE
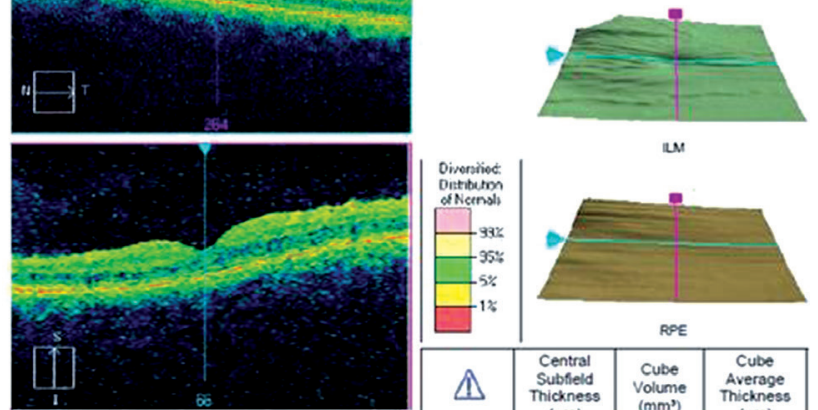

uM
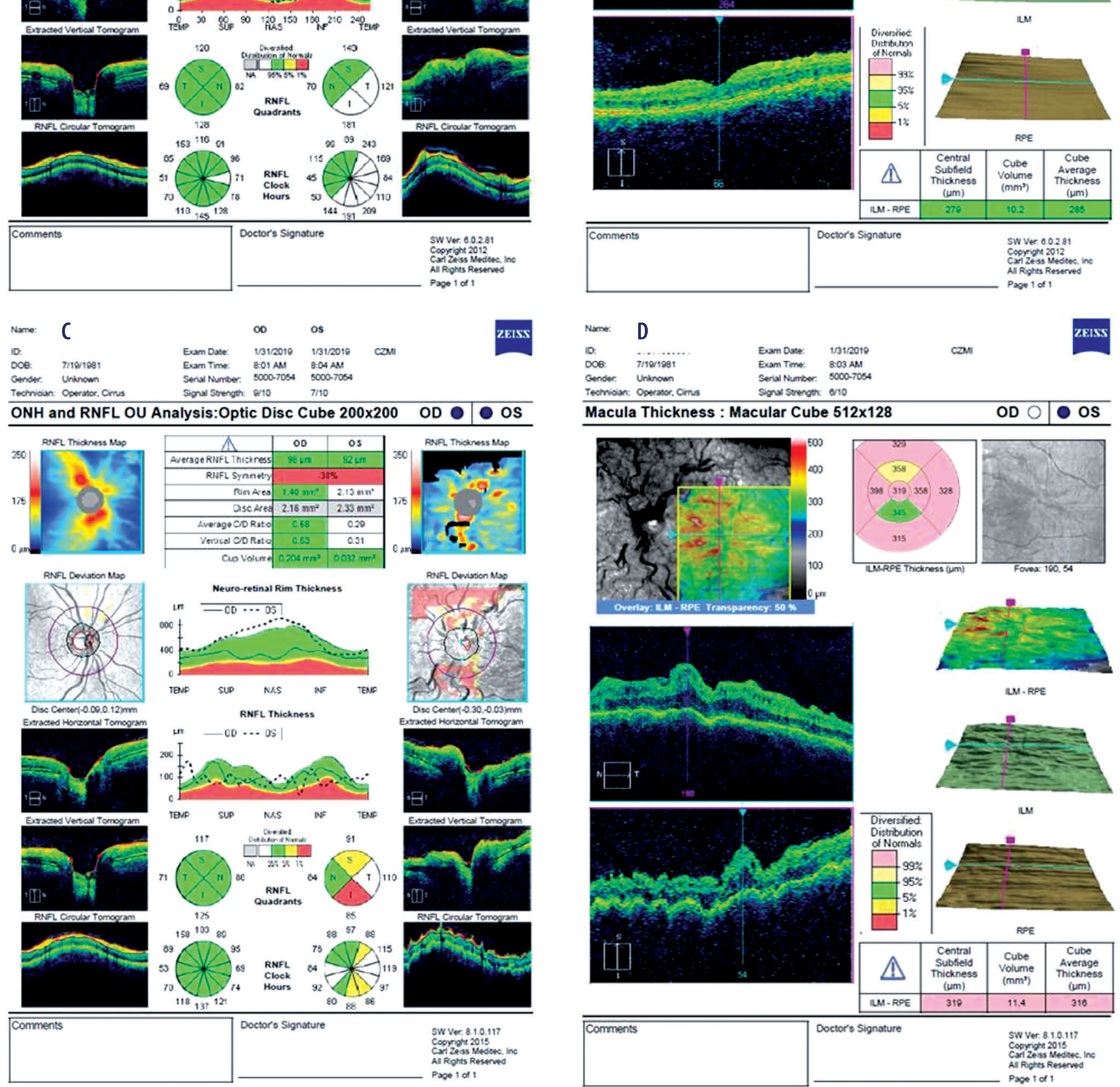

Figure 2. A) Optical coherence tomography (OCT) of the optic nerve disk at the time of diagnosis - swelling of the optic disc, significant thickening of the retinal nerve fibre layer (RNFL). B) OCT of macula at the time of diagnosis - retinal folds in the nasal part of the macula. C) OCT of the optic nerve disk after trabeculectomy - swelling of the optic nerve head with simultaneous normalization of the RNFL thickness. D) OCT of macula after trabeculectomy - retinal and choroidal folds 


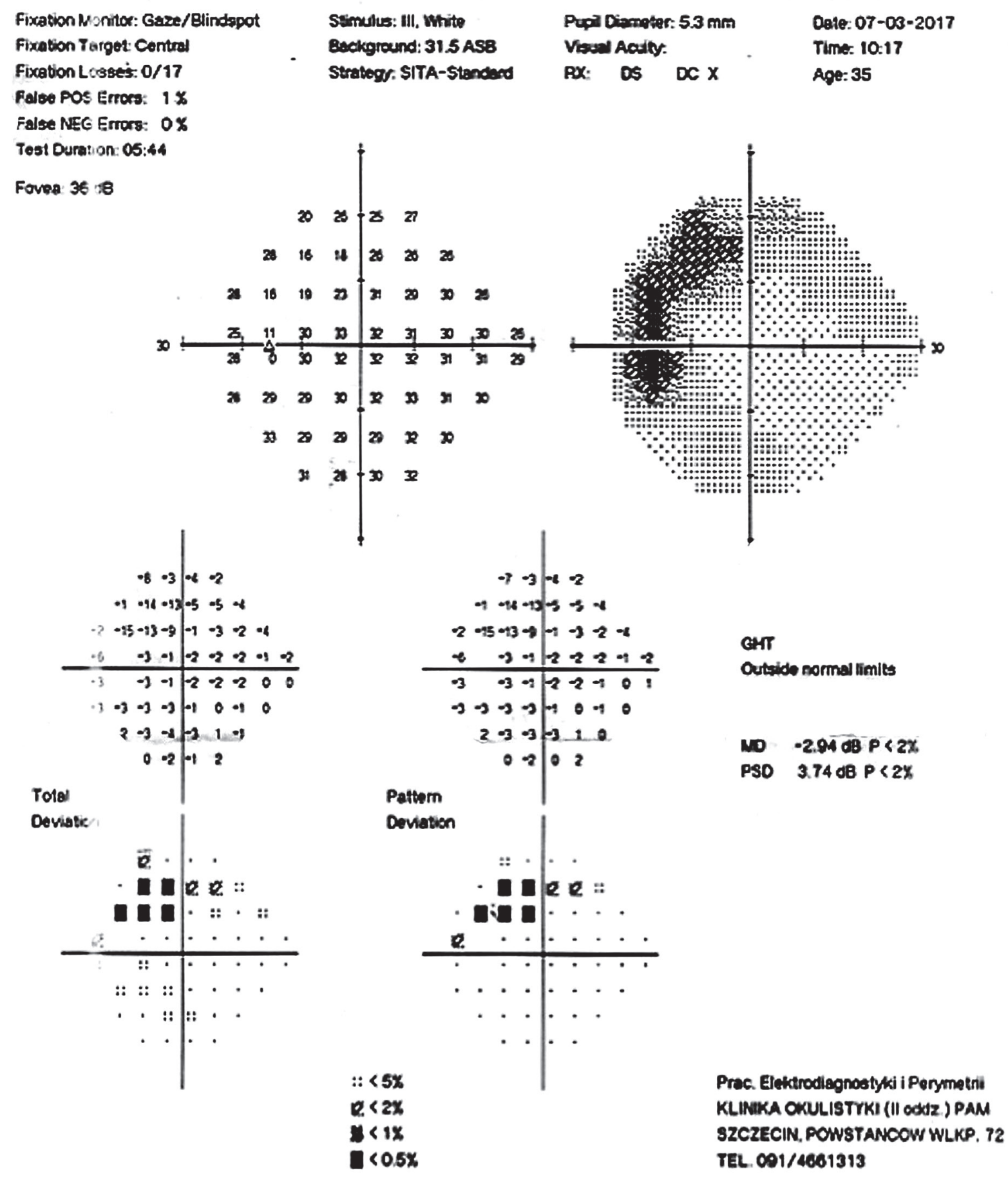

Figure 3. Statistical visual field (PS24/2, Humphrey) of left eye, before trabeculectomy - paracentral arcuate defect in upper hemisphere in left eye

Wilhelm [5], the most common visual field defects in primary ONSM include arcuate scotoma (29.9\%) and central scotoma (18.4\%), while according to Sibony they include peripheral constriction [7]. To date, only one case of concomitant ONSM and glaucoma has been described, where ONSM caused progression of the visual field defect despite normal IOP [7]. In the case in question, the patient had arcuate scotoma without features of progression in 3-year observation. It is hard to determine whether the visual field defect resulted from glaucoma-induced lesions or the presence of ONSM. Optic disc oedema prevented reliable reference of the visual field defects to defects of neuroretinal fibres.
In the case in question, the primary therapeutic issue was associated with persistent hypotony symptoms following the trabeculectomy procedure. Hypotony is a condition of decreased IOP $<6.5 \mathrm{mmHg}$ ( 3 standard deviations below the normal value). Typical complications of hypotony include oedema of the optic disc and macula, scleral collapse with secondary reduction of axial length (AL), retinal and choroidal folds, venous stasis and choroidal effusion in the eye fundus. The primary cause of hypotony is fistula surgery due to glaucoma (1.3-18\%) [8]. In the Tube Versus Trabeculectomy Study almost $25 \%$ of patients after trabeculectomy had hypotony; of these, $3 \%$ had hypotony maculopathy (functional 

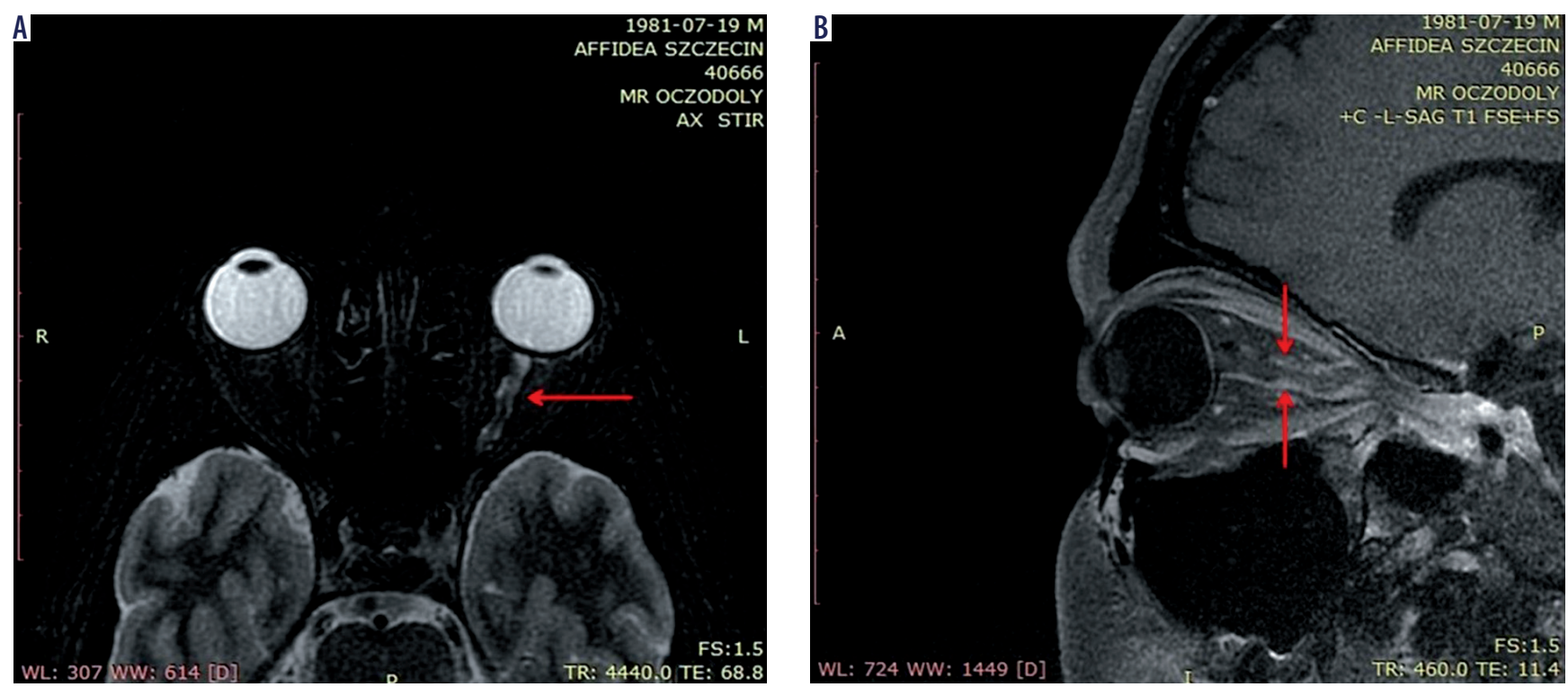

Figure 4. Orbit magnetic resonance imaging (MRI). Red arrow indicates thickened optic nerve sheath - suspicion of optic meningioma: A) axial projection, B) sagittal projection

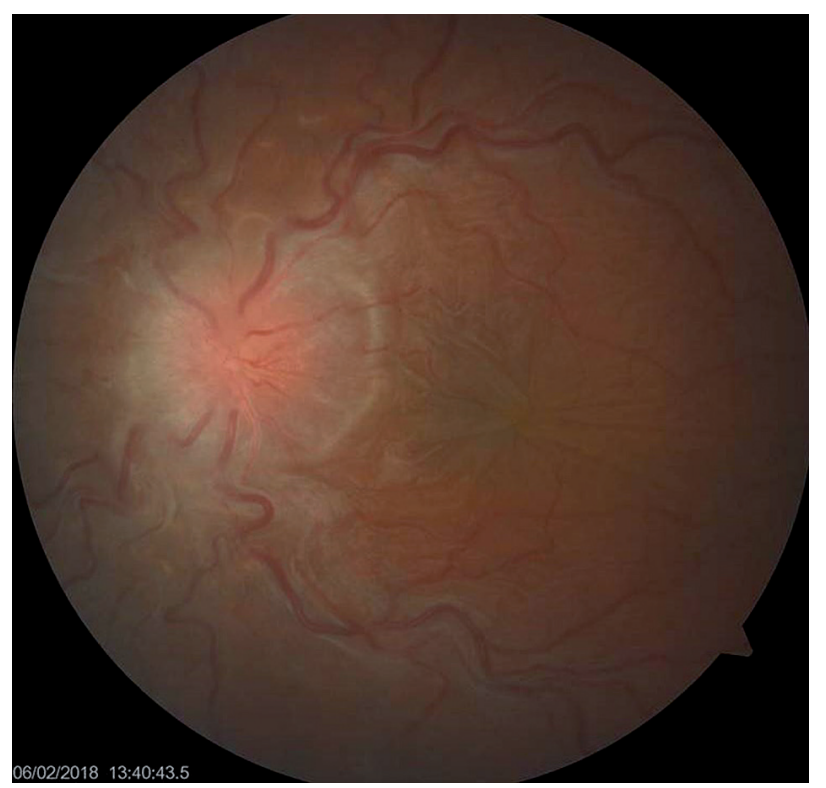

Figure 5. Image of the fundus after trabeculectomy - increased swelling of the optic disc, venous stasis, vein and artery tortuosity, retinal and choroidal fold

or anatomical macular damage) [9]. According to the most recent reports, the hypotony rate is lower, $1.7 \%$ [10], while the hypotony maculopathy rate is up to $0.2 \%$ [11]. The aetiology of vision deterioration in the course of hypotony is unclear. Most likely it may result from deformation of receptor lines due to retino-choroidal folds [12]. Risk factors of hypotony maculopathy include male gender, young age, use of antimetabolites (mitomycin is associated with higher risk than 5-fluorouracil), preoperative IOP increase, first anti-glaucoma surgery, and myopia. In the case in question, the patient had 6 of 7 risk factors (apart from myopia). To date, no literature reports on the potential effect of ONSM on hypotony maculopathy have been published.

Optic nerve sheath meningioma may cause retinal, choroidal and retino-choroidal folds. They are usually asymptomatic. The probable cause is compression of the posterior pole (directly by the tumour or oedematous optic disc) or choroidal effusion. In the case in question, the patient had asymptomatic small retinal folds accompanied with optic disc oedema in the preoperative period. After the trabeculectomy procedure, both massive retinal and choroidal folds, and choroidal effusion were found in the patient. Blood stasis in the choroid in the course of hypotony is known to have a hydrodynamic pathogenesis and results from the increase of filtration gradient and accumulation of proteins in the suprachoroidal space. However, the stasis symptoms did not resolve despite normalisation. The role of ONSM in this process is unclear. It is possible that ONSM reduces venous outflow from the choroid due to the mass effect or increased venous pressure in the orbit. Choroidal venous blood is drained to vorticose veins and then to superior and inferior ophthalmic veins. The superior ophthalmic vein is located between the optic nerve and superior rectus muscle. In this site, compression by the ONSM and obstruction of venous outflow from the eyeball is possible. Undoubtedly, the hypothesis warrants verification on a larger patient group. To date, no reports concerning the potential effect of ONSM on venous stasis or choroidal effusion syndrome have been published.

\section{CONCLUSIONS}

Optic nerve sheath meningioma may pass undetected in imaging examinations (CT, MRI, angioMRI) for a long time and mimic glaucoma. ONSM may potentially enhance choroidal effusion. The trabeculectomy procedure in patients with ONSM seems to be increased-risk surgery and therefore is not recommended. In such patients procedures associated with lower risk of post-operative hypotony (minimally invasive glaucoma surgery (MIGS), valves) should be considered.

\section{DISCLOSURE}

The authors declare no conflict of interest. 


\section{References}

1. Shapey J, Sabin HI, Danesh-Mayer HV, et al. Diagnosis and management of optic nerve sheath meningiomas. J Clin Neurosci 2013; 20: 1045-1056.

2. Basic and Clinical Science Series. Neuro-Ophthalmology. American Academy of Ophthalmology, 2014-2015.

3. Dorsey JF, Hollander AB, Alonso-Basanta M, et al. Cancer of the central nervous system. In: Niederhuber JE, Armitage J0, Doroshow JH, et al. Abeloff's Clinical Oncology. Elsevier Inc., Philadelphia, PA 2014; 938-1001.

4. Hokazono K, Moura FC, Monteiro ML. Optic nervemeningiomamimicking progression of glaucomatous axonal damage: a case report. Arq Bras Oftalmol 2008; 71: 725-728.

5. Wilhelm H, Dörr S, Paulsen F, et al. Early symptoms and findings in optic nerve meningiomas. Klin Monbl Augenheilkd 2009; 226: 869-874.

6. Dutton JJ. Optic nerve sheath meningiomas. Surv Ophthalmol 1992; 37: 167-183.

7. Sibony PA, Krauss HR, Kennerdell JS, et al. Optic nerve sheath meningiomas. Clinical manifestations. Ophthalmology 1984; 91: 1313-1326.

8. Fannin LA, Schiffman JC, Budenz DL. Risk factors for hypotony maculopathy. Ophthalmology 2003; 110: 1185-1191.

9. Gedde SJ, Schiffman JC, FeuerWJ, et al.; Tube versus Trabeculectomy Study Group. Treatment outcomes in the Tube Versus Trabeculectomy (TVT) study after five years of follow-up. Am J Ophthalmol 2012; 153: 789-803.

10. Tseng VL, Kim CH, Romero PT, et al. Risk factors and long-term outcomes in patients with low intraocular pressure after trabeculectomy. Ophthalmology 2017; 124: 1457-1465.

11. Khaw PT, Chiang M, Shah P, et al. Enhanced trabeculectomy: the Moorfields Safer Surgery System. Dev Ophthalmol 2012; 50: 1-28.

12. Costa VP, Arcieri ES. Hypotony maculopathy. Acta Ophthalmol Scand 2007; 85: 586-597. 